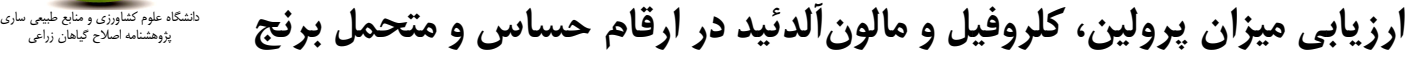
تحت تنث كلريدسديم مالون الديد در

\author{
زهرا سادات موسوىزاده'، حميد نجفى زرينى'، سيد حميدرضا هاشمى بطرودى ' و سيد كمال كاظمى تبارع \\ ا - دانش آموخته كارشناسى ارشد، دانشكده علوم زراعى، دانشخاه علوم كشاورزى و منابع طبيعى سارى

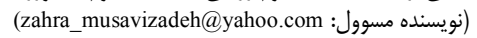

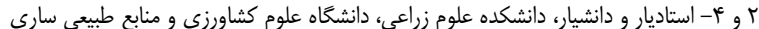

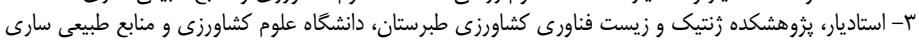

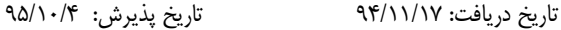

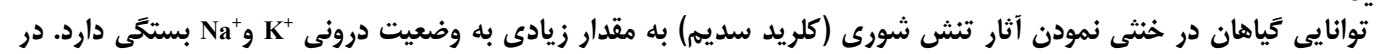

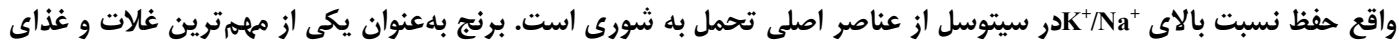

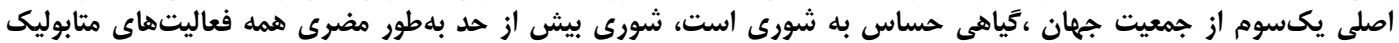

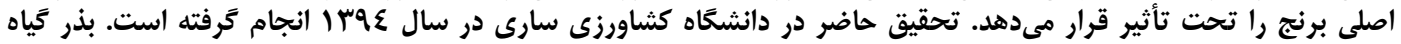

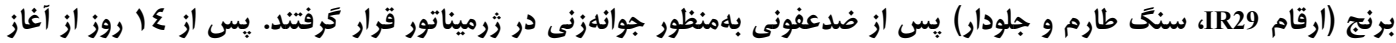

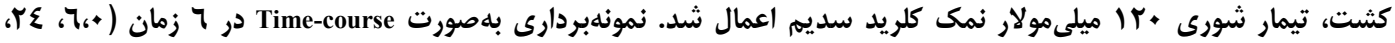

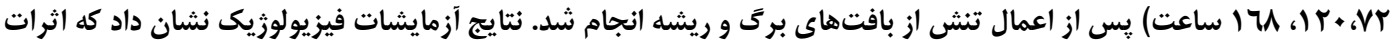

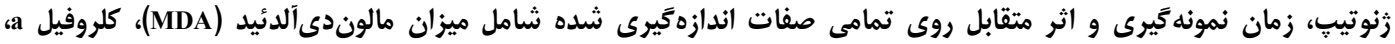

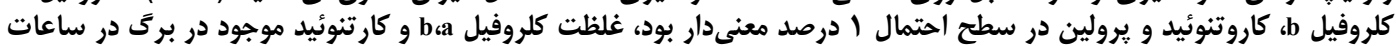

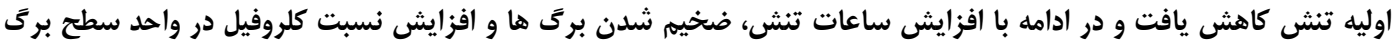

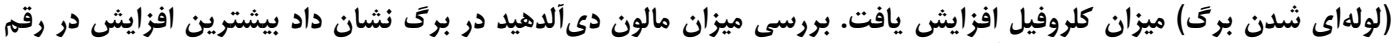

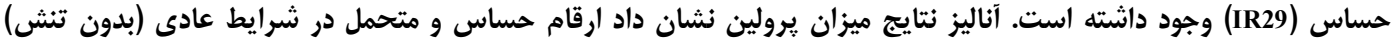

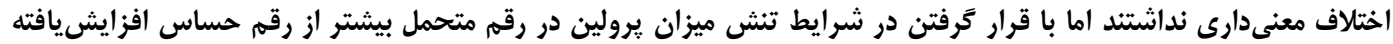

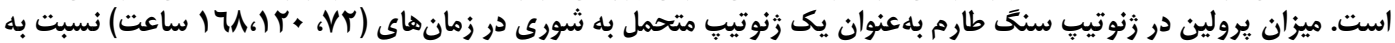

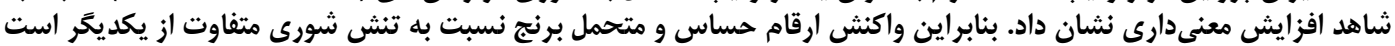

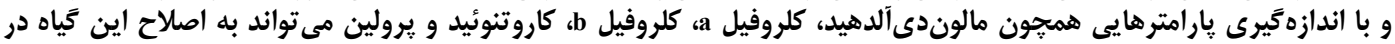
جهت تحمل به شورى كمك شايانى نمايد.

وازههاى كليدى: برنج، تنش كلريد سديم ، يرولين، كلروفيل، مالون آلدهيد

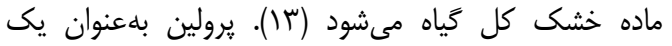

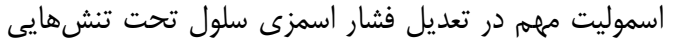

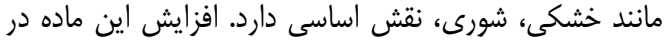

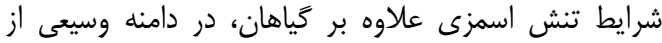

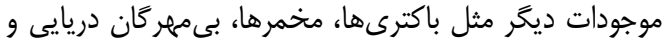

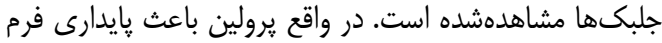

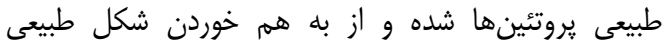

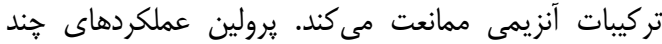

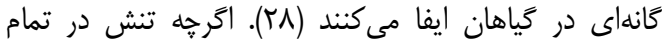

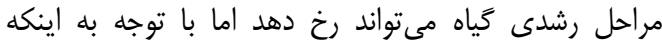

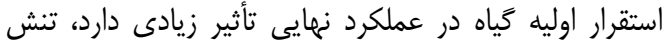

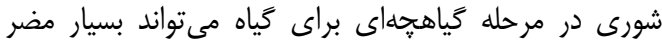

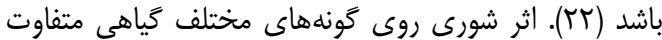

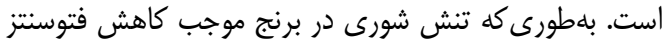

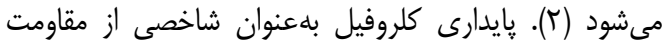

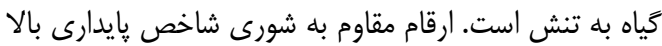

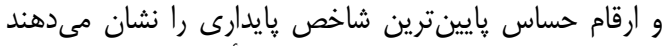

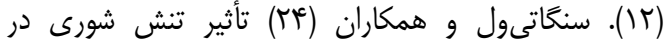

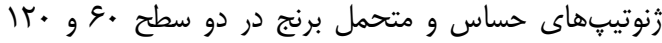

تنش شورى يكى از معضلات رو به گسترش است كه

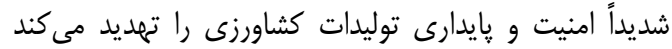
فئ)

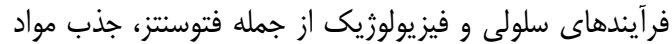

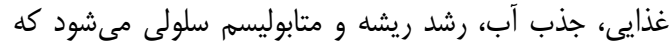

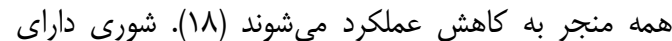

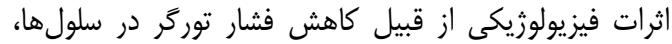

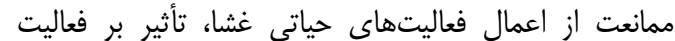

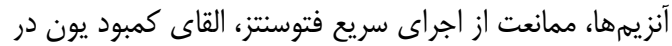

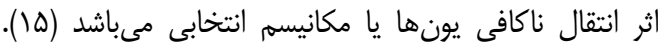

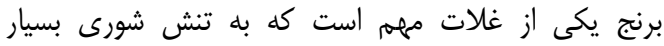

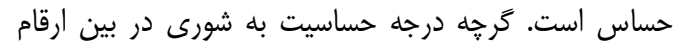

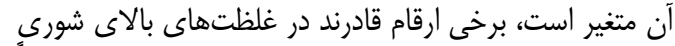

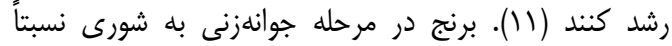

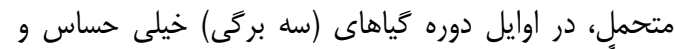

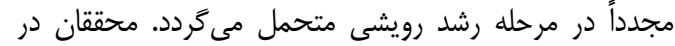

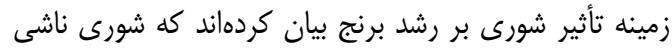

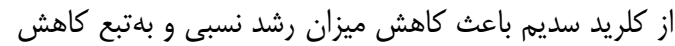


برنج سنگ طارم (متحمل به شورى)، رقم جلودار (نسبتاً

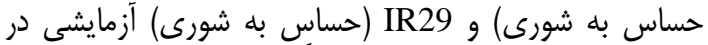

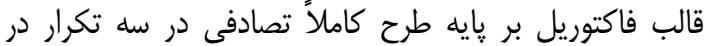

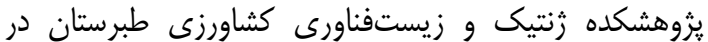

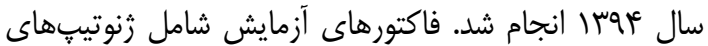

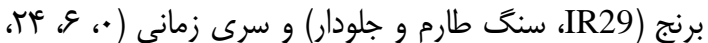

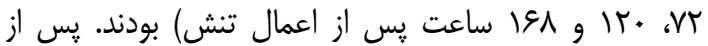

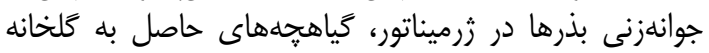

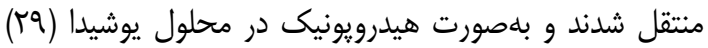

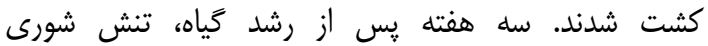

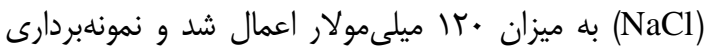

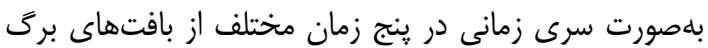

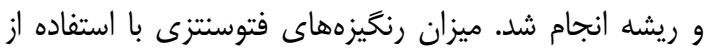

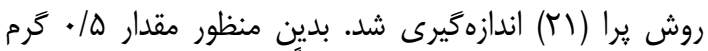

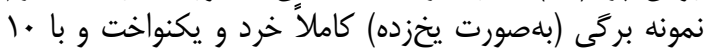

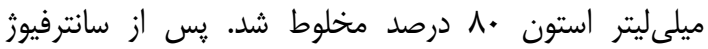

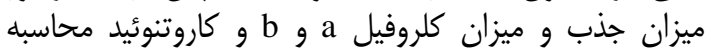

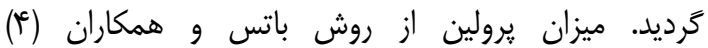

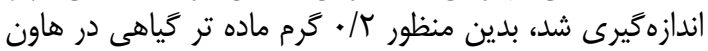

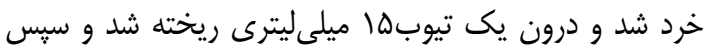

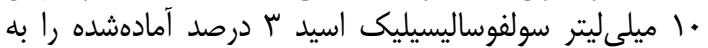

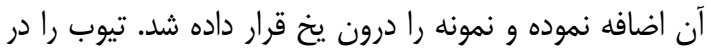

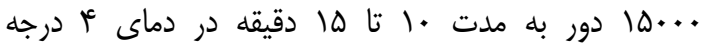

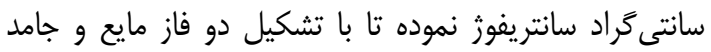

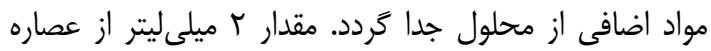

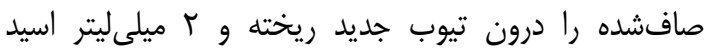

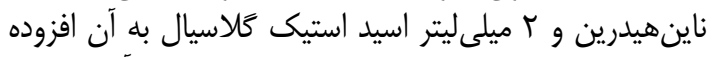

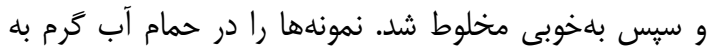

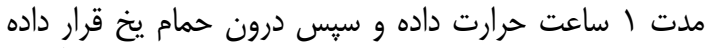

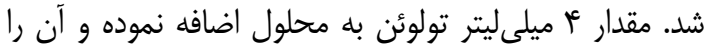

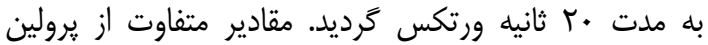

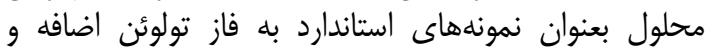

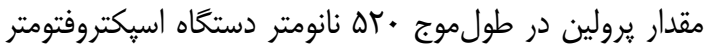

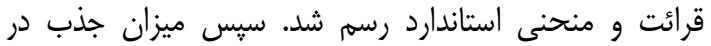

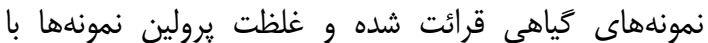

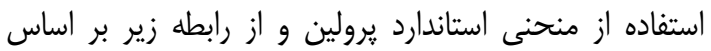

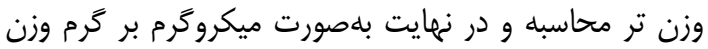
تر ارائه ترديد.

Proline $(\mu \mathrm{M} . \mathrm{g}-1$ fresh wt. $)=\frac{\mathrm{M \times T \times W}}{115.5} \times 1000$

كه در آن M: عدد قرائتشده با دستخاه اسبكتروفتومتر، T:

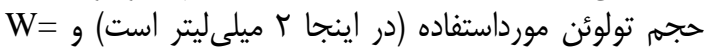

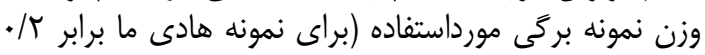

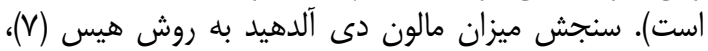

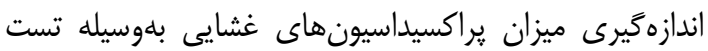

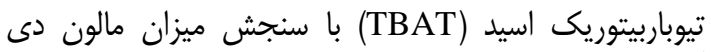

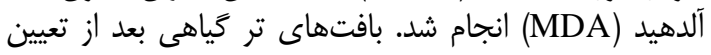

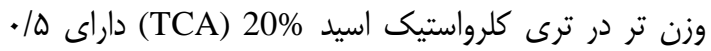
درصد تيوباربيتوريك اسيد همكن شده و بله مدت • ب دقيقه در
ميلىمولار مورد مطالعه قرار دادند. نتايج نشان داد سرعت

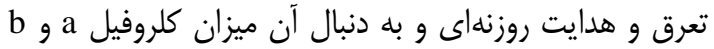

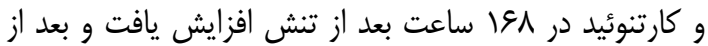

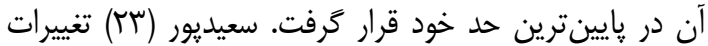

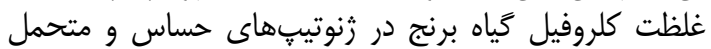

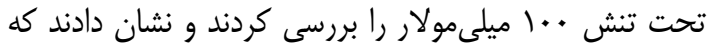

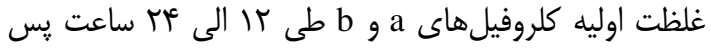

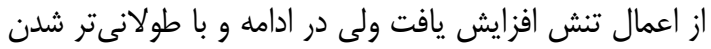

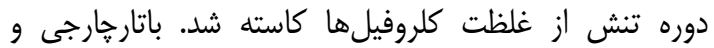

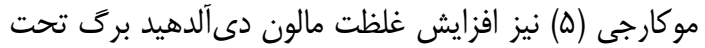

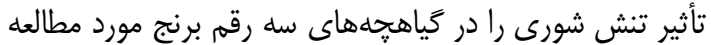

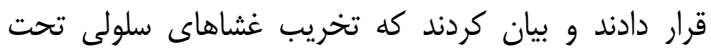

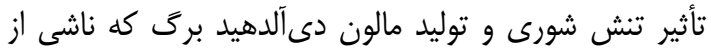

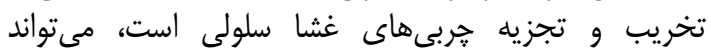

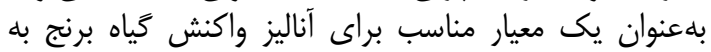

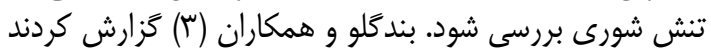

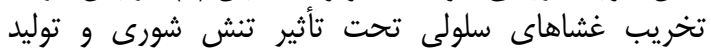

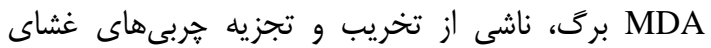

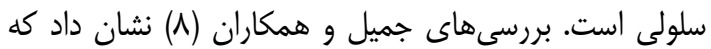

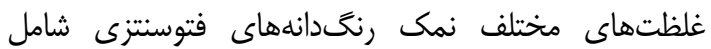

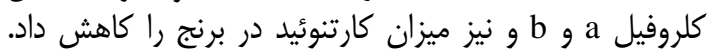

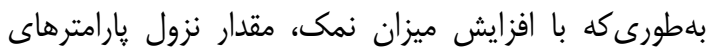

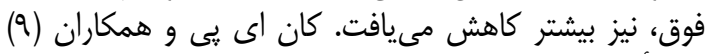

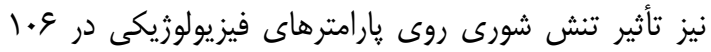

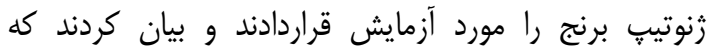

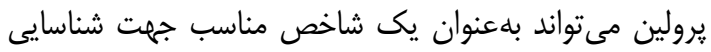

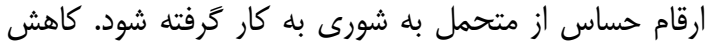

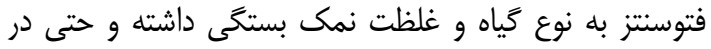

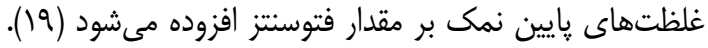

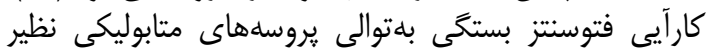
واكنشهاى فتوشيميايى، آنزيهماى دخيلى دخيل در تثبييت كربن،

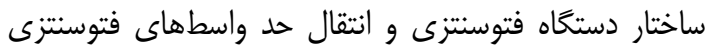

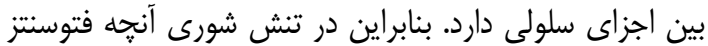

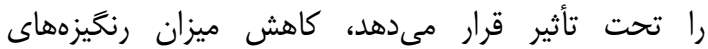
فتوسنتزى، كاهش سطح بركى (كاهش سطح فتر فترسنتزى)، كاهش فراهمى

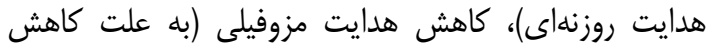
نفوذيذيرى غشا به

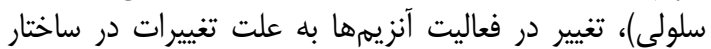

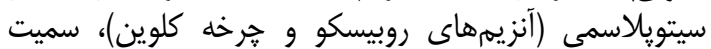
نمك، افزايش ييرى القاشده توسط شورى و آسيب اكسيدانياتيو به غشاهاى فتوسنتزى مى باشد (10).

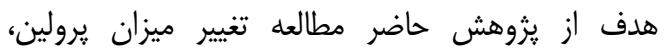
كلروفيل a a a م مالون آلدهيد در برخى از از ارقام برنج حساس و متحمل به شورى تحت تنش كلريدسديم مى مباشد.

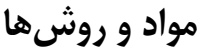

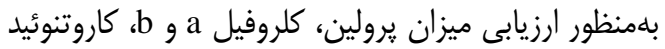

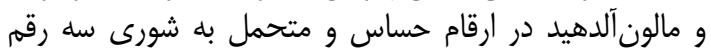




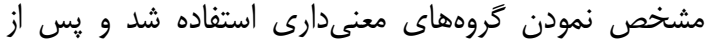

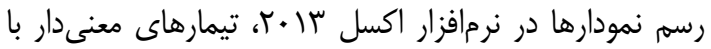
حروف غيرمشابه مشخص شدرار ندار اكند

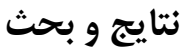

نتايج جدول تجزيه واريانس (جدول () نشان داد كه اثرات

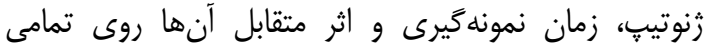

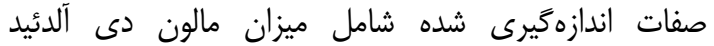

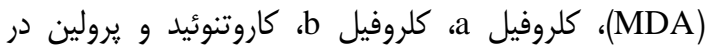
سطح احتمال الدرصد معنىدار بود.

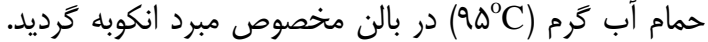

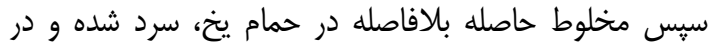

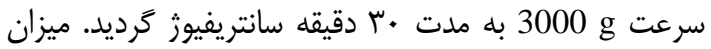

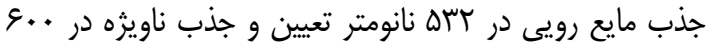

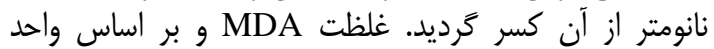

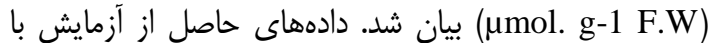
استفاده از نرمافزار SAS نسخه

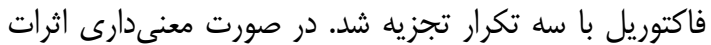

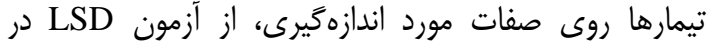

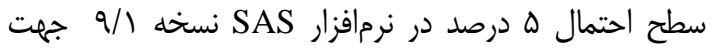

جدول ا- تجزيه واريانس (ميانخين مربعات) شاخص هاى فيزيولوزيك ارقام برنج تحت تنش شورى Table 1. Analysis of variance (mean square) of physiological indices of rice cultivars under salinity stress

\begin{tabular}{|c|c|c|c|c|c|c|}
\hline يرولين & كاروتنوئيد & كلروفيل b & a كلروفيل a & MDA & df & منابع تغييرات \\
\hline$. \ldots+\mu^{* *}$ & 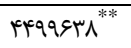 & $s \vee g / V^{* * *}$ & $r \Delta F / \Lambda^{* *}$ & $\mid \Delta \& \varepsilon \cdot 10^{* *}$ & r & زنوتيب \\
\hline$\cdot / \cdots 10^{* * *}$ & $r \cdot 94 \cdot 1)^{* *}$ & $10 \cdot 9 / 9^{* *}$ & $V N / \Lambda^{* * *}$ & $r q \cdot \vee /\left.\right|^{* *}$ & $\Delta$ & زمان \\
\hline$\cdot / \cdots \mid r^{* * *}$ & $199 . .9^{* *}$ & $|\Delta \Psi /|^{* *}$ & $r q / q^{* *}$ & 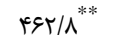 & 1. & زنوتيّ × زمان \\
\hline.$|\ldots \ldots|$ & $T V \cdot T$ & $N / T$ & $ه /$. & Q DI/II & ع & خطا \\
\hline$\cdot / V^{c}$ & $T / V)$ & $11 / 1$ & $1 \% \%$ & $1 \pi / 0$ & & ضريب تغييرات (٪) \\
\hline
\end{tabular}

(شكل () .در رقم IR29 و سنگ طارم بلهور كلى با افزايش

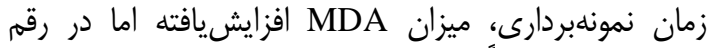

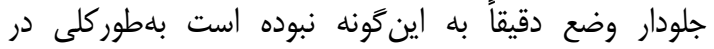
زمانهاى بالاتر، ميزان MDA بالاتر بوده است (شكل ().

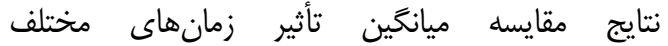

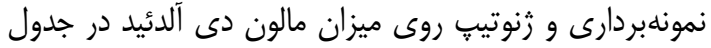
ا نشان داده شده است. بيشترين مقدار

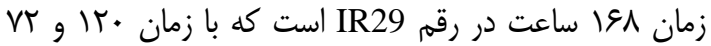

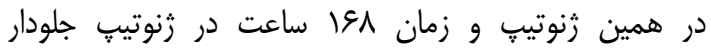
اختلاف آمارى معنىدارى از نظر آزمون LSD 5\% نداعت دراشت

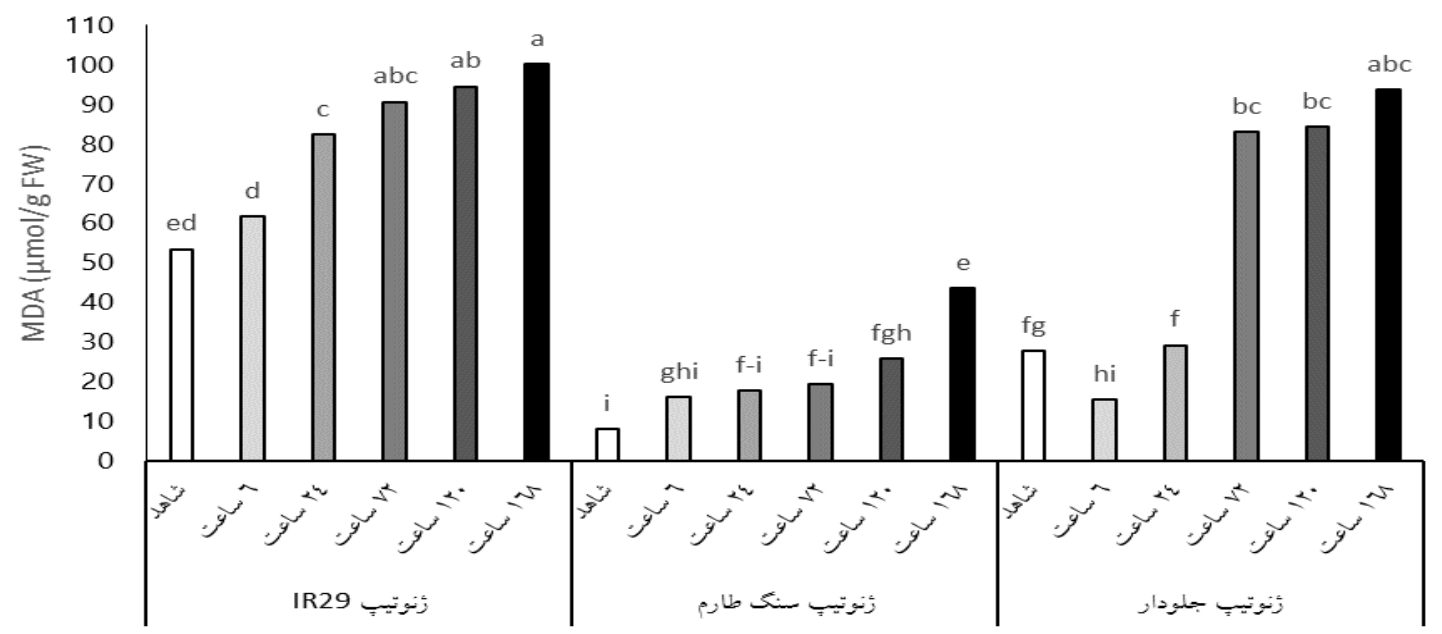

شكل 1- مقايسه ميانخين مالون دى آلدئيد

Figure 1. Comparison of mean of Malondialdehyde

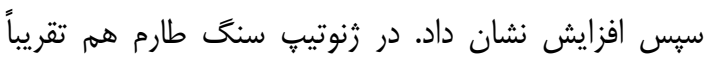

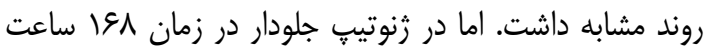

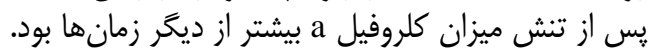

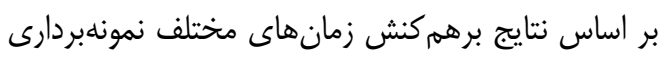

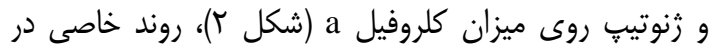

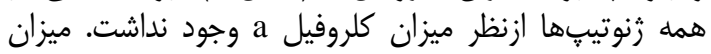
كلروفيل a در رقم IR29 با افزايش زمان ابتدا كاهش يافت و ونئ 


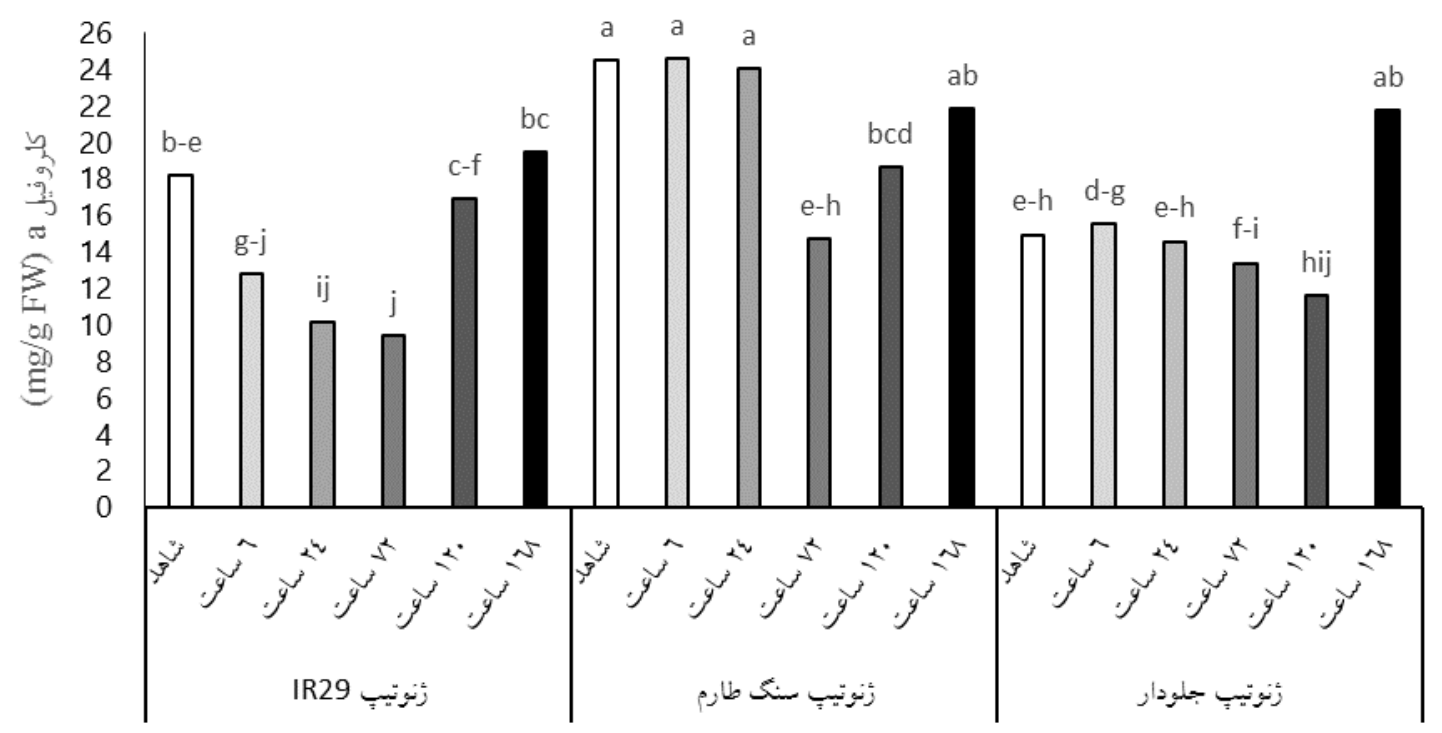

a شكل r- مقايسه ميانكين كلروفيل

Figure 2. Comparison of mean of Chlorophyll a

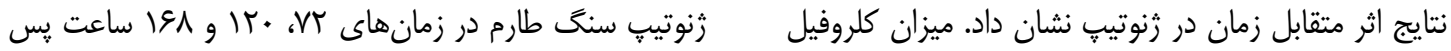

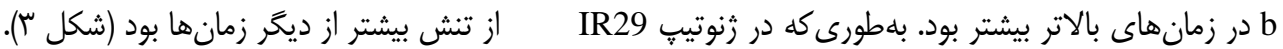

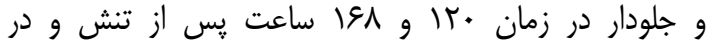

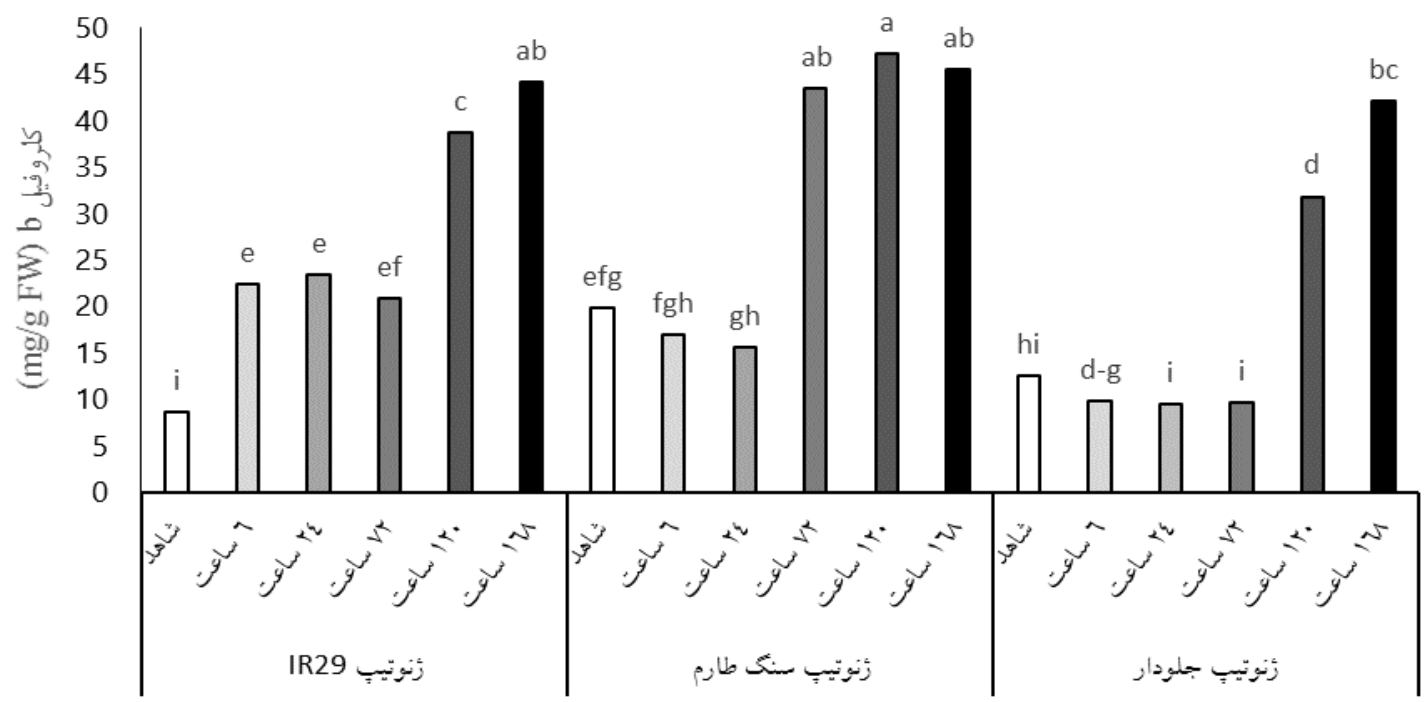

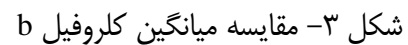

Figure 3. Comparison of mean of Chlorophyll b

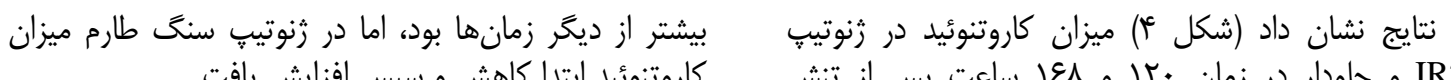

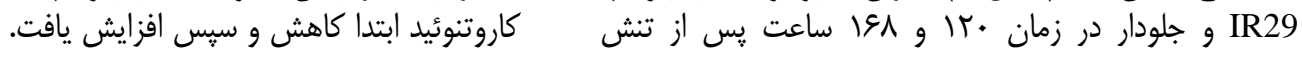




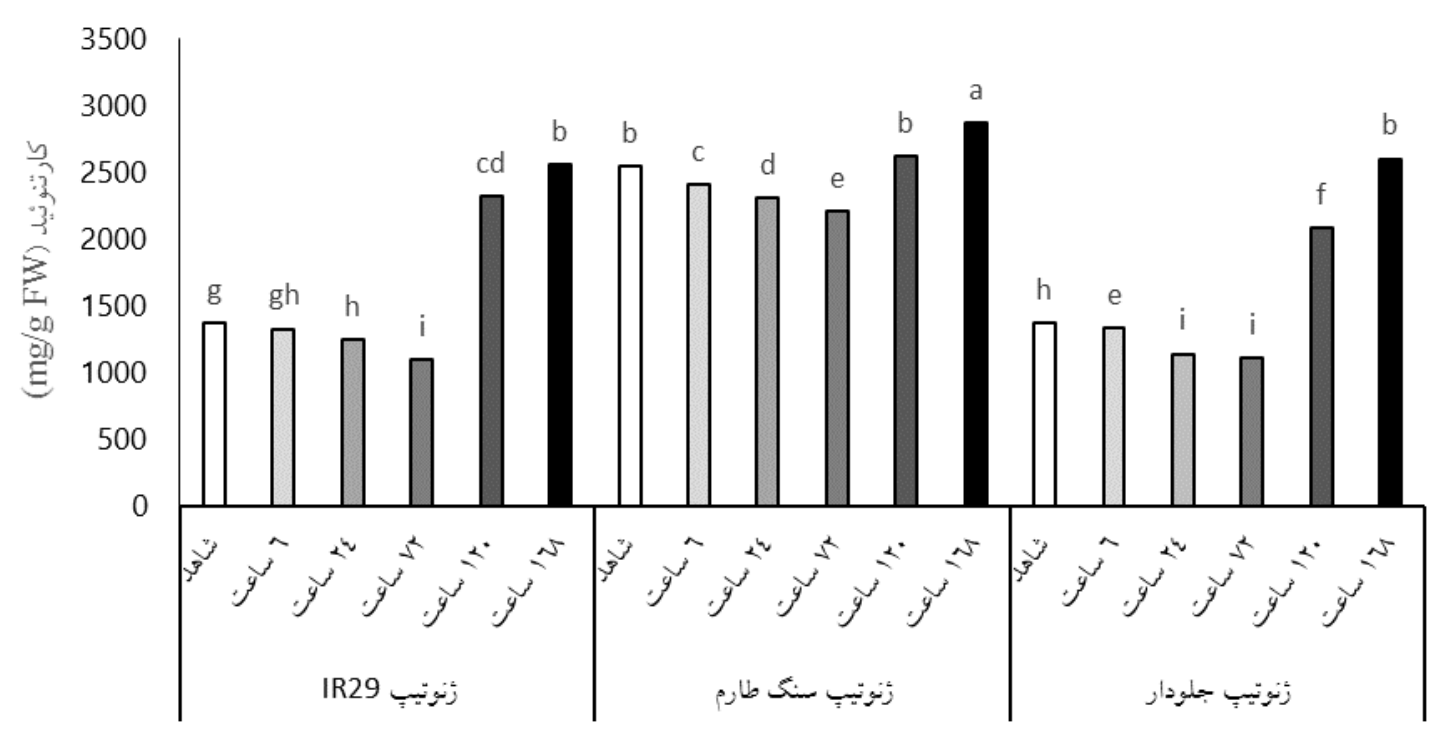

شكل r - مقايسه ميانكين كاروتنوئيد

Figure 4. Comparison of mean of Carotenoid

افزايش معنىدارى نسبت به تيمار شاهد داشت اما اين تغيير

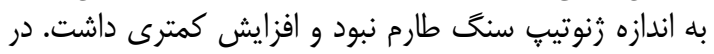

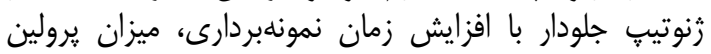

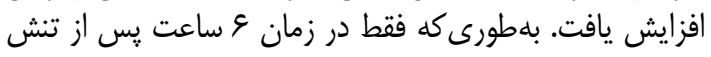

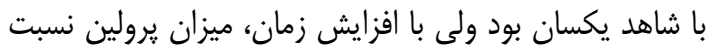
به شاهد افزايش بيشترى يافت.

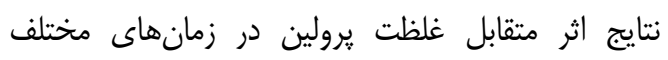

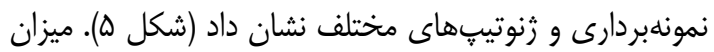

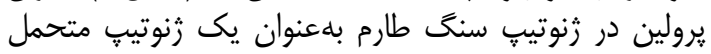

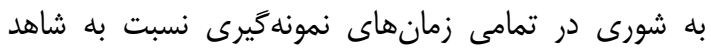

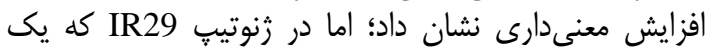

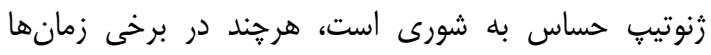

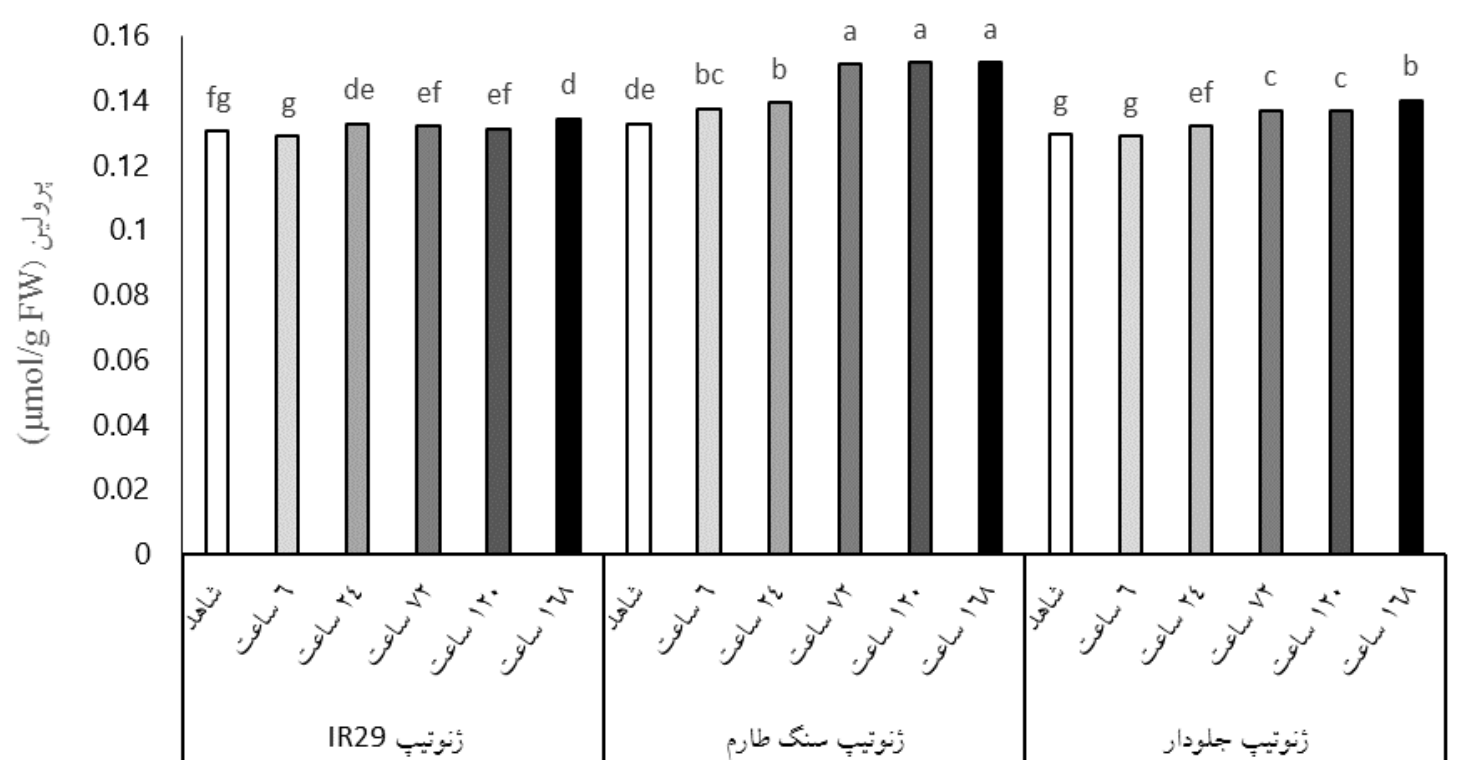

شكل ه- مقايسه ميانگين يرولين

Figure 5. Comparison of mean of Proline 
از اعمال تنش افزايش يافت كه حاكى از بالا بودن غلظت

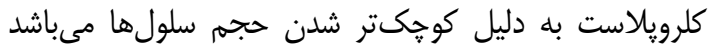

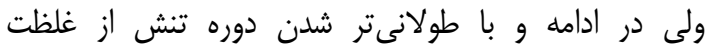

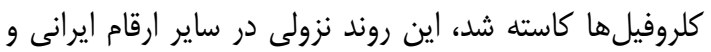

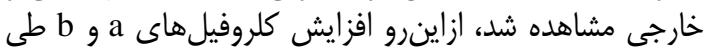

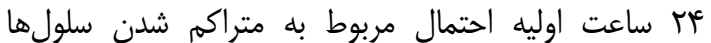

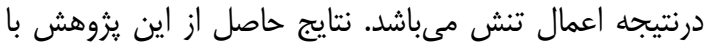

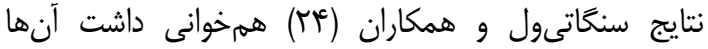

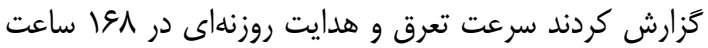

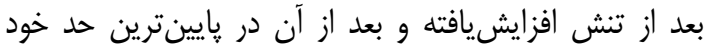

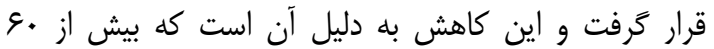

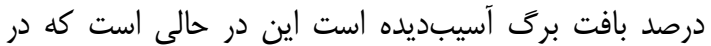

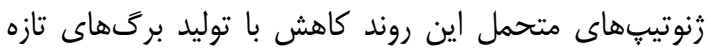

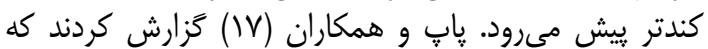

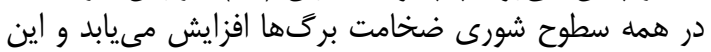

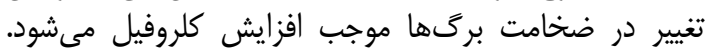

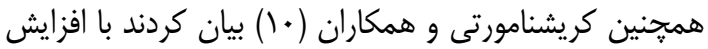

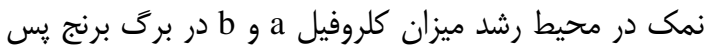

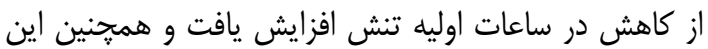

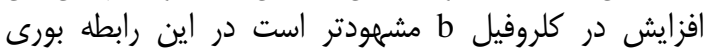

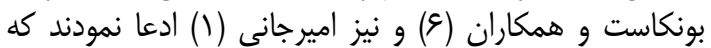

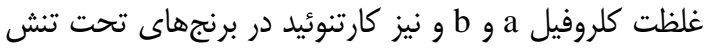

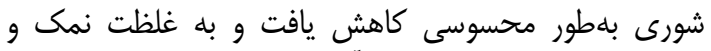

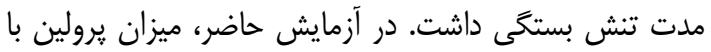

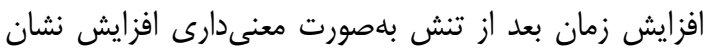

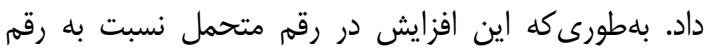

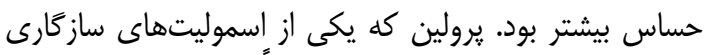

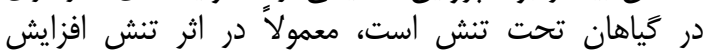

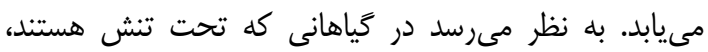

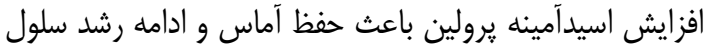

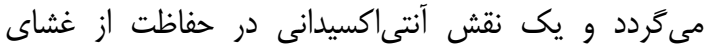

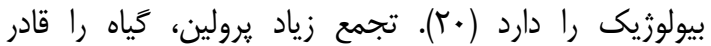

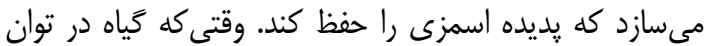

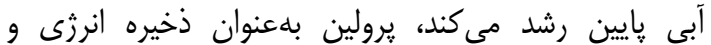

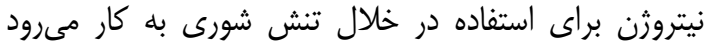

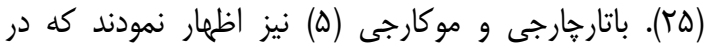

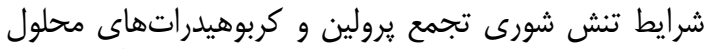

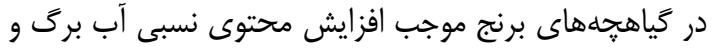

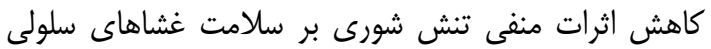

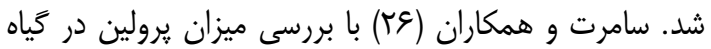

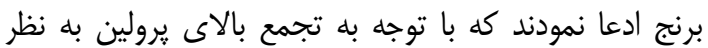

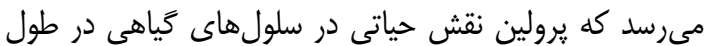
تنش شورى ايفا مى كند.

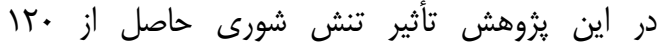

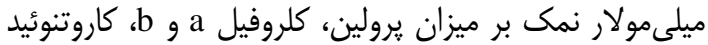

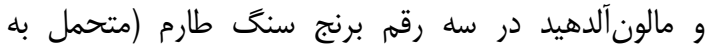

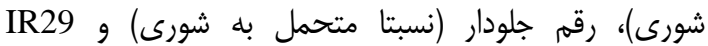

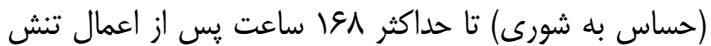

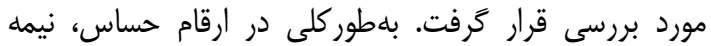

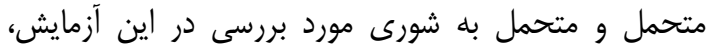

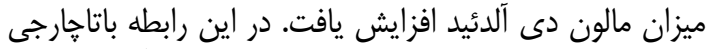

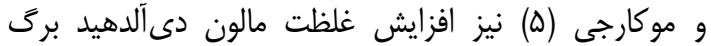

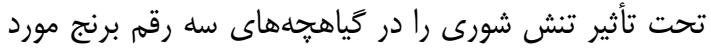

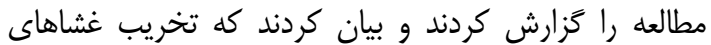

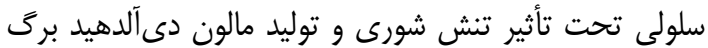

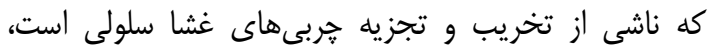

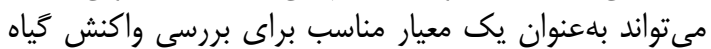

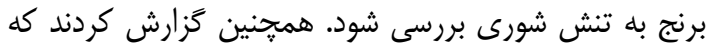

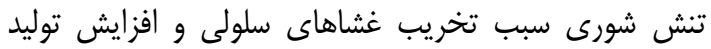

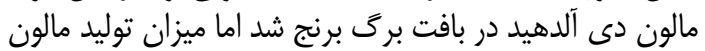

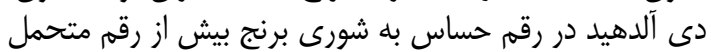

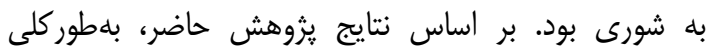

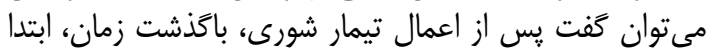

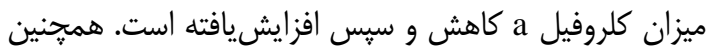

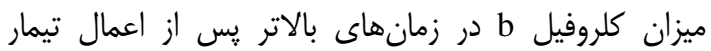

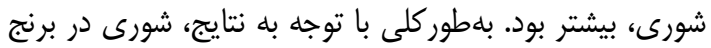

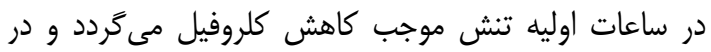

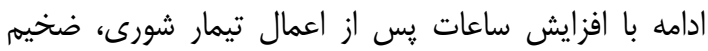

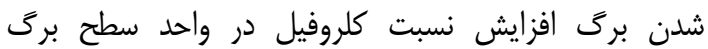

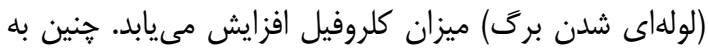

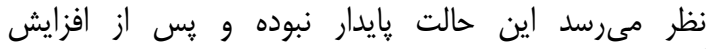

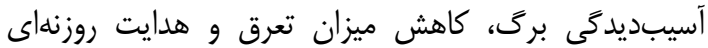

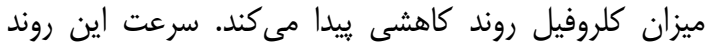

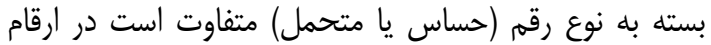

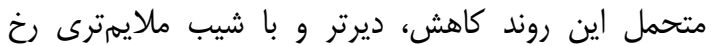

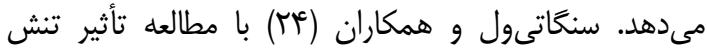

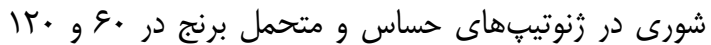

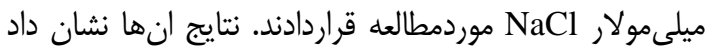

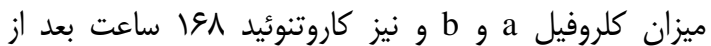

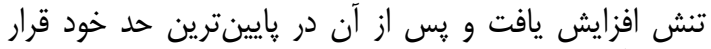

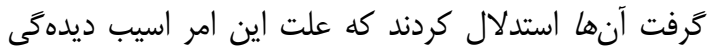

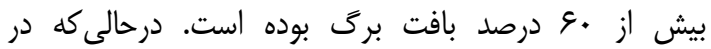

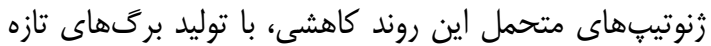

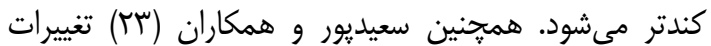

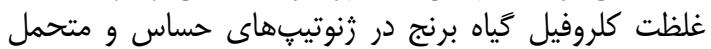

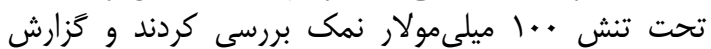

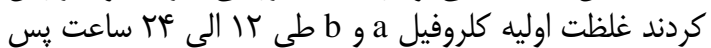


1. Amirjani Amirjani, M.R. 2011. Effect of salinity stress on growth, sugar content, pigments and enzyme activity of
rice. Int J Bot, 7: 73-81 (In Persian).

2. Asch, F., M. Dingkuhn and K. Dorffling. 2000. Salinity increases CO2 assimilation but reduces growth in field-grown, irrigated rice. Plant and Soil, 218: 1-10.

3. Bandeoğlu, E., F. Eyidoğan, M. Yücel and H.A. Öktem. 2004. Antioxidant responses of shoots and roots of lentil to NaCl-salinity stress. Plant Growth Regulation, 42: 69-77.

4. Bates, L.S. 1973. Rapid determination of Free Proline for water- Stress studies. Plant and Soil, 39: $205-207$.

5. Bhattacharjee, S. and A.K. Mukherjee. 2002. Salt stress induced cytosolute accumulation, antioxidant response and membrane deterioration in three rice cultivars during early germination. Seed Science and Technology, 30: 279-287.

6. Boriboonkaset, T., C. Theerawitaya, N. Yamada, A. Pichakum, K. Supaibulwatana, S. Cha-um and C. Kirdmanee. 2013. Regulation of some carbohydrate metabolism-related genes, starch and soluble sugar contents, photosynthetic activities and yield attributes of two contrasting rice genotypes subjected to salt stress. Protoplasma, 250: 1157-1167.

7. Heath, R.L. and L. Packer. 1968. Photoperoxidation in isolated chloroplasts: I. Kinetics and stoichiometry of fatty acid peroxidation. Archives of biochemistry and biophysics, 125(1): 189-198.

8. Jamil, M., S.A. Bashir, M.I.N.A. Anwar, S.O.M.A. Bibi, S.A.S.I.A. Bangash, F. Ullah and E.S. Rha. 2012. Effect of salinity on physiological and biochemical characteristics of different varieties of rice. Pak $J$ Bot, 44: 7-13.

9. Kanawapee, N.J. Sanitchon, W. Lontom and P. Threerakulpisut. 2012. Evaluation of salt tolerance at the seedling stage in rice genotypes by growth performance, ion accumulation, proline and chlorophyll content. Plant and soil, 358: 235-249.

10. Krishnamurthy, R., M. Anbazhagan and K.A. Bhagwat. 1987. Effect of NaCl toxicity chlorophyll breakdown in rice. Indian Journal of Agricultural Sciences 57: 567-570.

11. Mekawy, A.M.M., D.V.M. Assaha, H. Yahagi, Y. Tada, A. Ueda and H. Saneoka. 2015. Growth, physiological adaptation, and gene expression analysis of two Egyptian rice cultivars under salt stress. Plant Physiology and Biochemistry, 87: 17-25.

12. Mohan, M.M., S.L. Narayanan and S.M. Ibrahim. 2000. Chlorophyll stability index (CSI): its impact on salt tolerance in rice. International Rice Research Notes, 25(2): 38-39.

13. Moradi, F. 2002. Physiological characterization of rice cultivars for salinity tolerance during vegetative and reproductive stages. Los Baños, Laguna, Philippines, 190 pp (In Persian).

14. Moumeni, A. 2011. Geographical distribution and salinity of soil resources in Iran. Iranian Journal of Soil Research, 24: 203- 215 (In Persian).

15. Munns, R., R.A. James and A. Läuchli. 2006. Approaches to increasing the salt tolerance of wheat and other cereals. Journal of experimental botany, 57(5): 1025-1043.

16. Orcutt, D.M. and E.T. Nilsen. 2000. The physiology of plants under stress: soil and biotic factors (Vol. 2). John Wiley \& Sons. New York, NY, USA. 684 pp.

17. Papp, J.C., M.C. Ball and N. Terry. 1983. A comparative study of the effects of $\mathrm{NaCl}$ salinity on respiration, photosynthesis, and leaf extension growth in Beta vulgaris L. (Sugar beet). Plant, Cell and Environment, 6: 675-677.

18. Pardo, J.M. 2010. Biotechnology of water and salinity stress tolerance. Curr. Opin. Biotech, 21: 185-196.

19. Parida, A.K. and A.B. Das. 2005. Salt tolerance and salinity effects on plants: a review. Ecotoxicology and environmental safety, 60: 324-349.

20. Patakas, A., N. Nikolaou, E. Zioziou, K. Radoglou and B. Noitsakis. 2002. The role of organic solute and ion accumulation in osmotic adjustment in drought-stressed grapevines. Plant Science, 163:361-367.

21. Porra, R.J. 2002. The chequered history of the development and use of simultaneous equations for the accurate determination of chlorophylls a and b. Photosynthesis Research, 73: 149-156.

22. Rauf, M., M. Munir, M. Hassan, M. Ahmad and M. Afzal. 2007. Performance of wheat genotypes under osmotic stress at germination and early seedling growth stage. African journal of biotechnology, $6-8$ pp.

23. Saeedpour, S. 2015. Effect of salinity on growth, chlorophyll content and ions uptake of rice cultivars (Oryza sativa) cultivars. Agronomy Journal (Pajouhesh and Sazandegi), 102: 2-11 (In Persian).

24. Senguttuvel, P., C. Vijayalakshmi, K. Thiyagarajan, J.R. Kannanbapu, S. Kota, G. Padmavathi and B.C. Viraktamath. 2014. Changes in photosynthesis, chlorophyll fluorescence, gas exchange parameters and osmotic potential to salt stress during early seedling stage in rice (Oryza sativa L). SABRAO Journal of Breeding and Genetics, 46: 120-135.

25. Sudhakar, C., P.S. Reddy and K. Veeranjaneyulu. 1993. Effect of salt stress on the enzymes of proline synthesis and oxidation in greengram (Phaseolus aureus Roxb.) seedlings. Journal of plant physiology, 141: 621-623.

26. Summart, J., P. Thanonkeo, S. Panichajakul, P. Prathepha and M.T. McManus. 2010. Effect of salt stress on growth, inorganic ion and proline accumulation in Thai aromatic rice, Khao Dawk Mali 105, callus culture. African Journal of Biotechnology, 9(2).

27. Wu, T., W. Lin Kao and C.H. Hong. 2015. Gene knockout of glutathione reductase 3 results in increased sensitivity to salt stress in rice. Plant Molecular Biology, Volume 87, Issue, 6: 555-564.

28. Yamchi, A., F.R. Jazii, C. Ghobadi, A. Mousavi and A.A. Karkhanehee. 2005. Increasing of tolerance to osmotic stresses in tobacco Nicotiana tabacum cv. xanthi through overexpression of p5cs gene. JWSSIsfahan University of Technology, 8: 31-40 (In Persian).

29. Yoshida, S., D.A. Forno, J.H. Cook and K.A. Gomez. 1976. Laboratory Manual for Physiological Studies of Rice. International Rice Research Institute, Manila, pp: 61-65. 


\title{
Assessment of Proline, Chlorophyll and Malondialdehyde in Sensitive and Tolerant Rice (Oryza sativa L.) Cultivars under Salt Stress Conditions
}

\author{
Zahra Sadat Musavizadeh ${ }^{1}$, Hamid Najafi Zarini ${ }^{2}$, Seyyed Hamidreza Hashemi-Petroudi ${ }^{3}$ \\ and Seyyed Kamal Kazemitabar ${ }^{4}$ \\ 1- Graduated M.Sc. Student, Department of Agricultural Sciences, Sari Agricultural Sciences and Natural Resources \\ University (Corresponding Author: zahra_musavizadeh@yahoo.com) \\ 2 and 4- Assistant Professors and Associated Professors, Department of Agricultural Sciences, Sari Agricultural \\ Sciences and Natural Resources University \\ 3- Assistant Professors, Genetic and Agricultural Biotechnology Institute of Tabarestan (GABIT), Sari Agricultural \\ Sciences and Natural Resources University \\ Receive: January 7, $2016 \quad$ Accepted: December 24, 2016
}

\begin{abstract}
Salinity stress is one of the main obstacles in crop production in many parts of the world, especially in arid and semi-arid regions. Plants' ability to neutralize the effect of salinity largely depends on the internal state $\mathrm{K}^{+}$and $\mathrm{Na}^{+}$. In fact, maintaining high $\mathrm{K}^{+} / \mathrm{Na}^{+}$ratio in Cytosol is a key element for tolerance to salinity. Rice, one of the most important food crops, a primary food source for more than one-third of the world's population. Rice is sensitive to salinity stress, so that, salt effects will extensively damage rice metabolic activities. This study has presented the effects of salinity on some physiological characteristics and genes expression patterns that coded for a number of ion channels in rice. In order to seed germination, the seeds of some cultivar (IR29, Sangtarom, and Jelodar) sterilized and then were placed in germinator. Salinity treatment $(120 \mathrm{mM} \mathrm{NaCl})$ were applied 14 days after culture. The leaf and root samples were collected at six time-courses $(0,6,24,72,120$ and 168 hours) after sodium chloride stress. Results of physiological assessments showed that the effects of genotype, sampling period and their interactions were significant at $1 \%$ level of probability on all measured traits including malondialdehyde (MDA), chlorophyll a, chlorophyll b, carotenoids and proline contents. The concentration of chlorophyll a, b and carotenoid of leaves were reduced in early hours of encountering with stress and then increasing the hours of stress, leaf thickness (leaf rolling) caused chlorophyll per leaf unit and increases the amount of chlorophyll. The measurement of MDA in the leaves showed that the greatest increase occurred in sensitive cultivar (IR29). Proline content analysis showed that there was no significant difference between proline content of sensitive and tolerant cultivars under normal condition (no stress), but by exposure to salinity stress, proline content was increased in susceptible more than tolerant cultivar. Proline content in Sangtaroom, as a salt tolerant genotype, has significantly increased in comparison with the control cultivar in 72,120 and 168 (h) times. Consequently, the responses of sensitive and tolerant rice cultivars to the salinity stress are different from each other and measuring of some parameters such as MDA, chlorophyll a, chlorophyll $b$, carotenoids, and proline can help to improve the salt tolerance of plants.
\end{abstract}

Keywords: Chlorophyll, Malondialdehyde, $\mathrm{NaCl}$ stress, Proline, Rice 